\title{
25 Research Square \\ Bendamustine Plus Rituximab Versus R-Minichop as First-Line Treatment in Unfit Patients with Diffuse Large B-Cell Lymphoma
}

\section{Dongdong Zhang}

Hubei University of Medicine https://orcid.org/0000-0001-7611-3114

Tao Liu

Huazhong University of Science and Technology Tongji Medical College

Natasha Kaweme

Wuhan University

Youhong Dong ( $\nabla$ dongyouhong2005@163.com )

xiangyangshi diyi renmin yiyuan: Xiangyang No 1 People's Hospital Affiliated to Hubei University of Medicine

\section{Liling Zhang}

Huazhong University of Science and Technology Tongji Medical College

\section{Research Article}

Keywords: Diffuse large-B cell lymphoma, Bendamustine plus rituximab, R-miniCHOP, Unfit, Objective remission rate.

Posted Date: June 28th, 2021

DOl: https://doi.org/10.21203/rs.3.rs-580186/v1

License: @ (i) This work is licensed under a Creative Commons Attribution 4.0 International License. Read Full License 


\section{Abstract}

Objective: To evaluate the efficacy and safety of bendamustine plus rituximab (BR) and low-does CHOP chemotherapy regimen-rituximab combination (R-miniCHOP) in elderly patients with diffuse large- $\mathrm{B}$ cell lymphoma, unfit for full dose immunochemotherapy.

Method: Patients over 70 years with diffuse larger B-cell lymphoma or follicular lymphoma grade 3B, defined as unfit according to Comprehensive Geriatric Assessment (CGA), were included in our study. BR or R-miniCHOP was individualized according to the patients' performance status and the presence of comorbidities. All the patients were received 4-6 cycles of immunochemotherapy at three-week intervals. Objective remission rate and adverse reaction were evaluated between the two groups.

Result: This study included thirty-two patients enrolled between January 2020 and November 2020. The median age was 74 years (range 70-82). Twelve patients received BR, whereas twenty patients were treated with R-miniCHOP. The objective remission rate in the BR group was a slightly higher than the RminiCHOP group ( $83.3 \%$ vs $75 \%)$, but with no statistical significance $(P=0.581)$. Furthermore, the objective remission rate was influenced by a serum beta 2 microglobulin concentration of $3 \mathrm{mg} / \mathrm{L}$ or less. The BR group showed a lower incidence of leukopenia than the R-miniCHOP group $(P=0.029)$, but had a higher incidence of fever $(P=0.036)$.

Conclusion: BR showed equivalent efficacy but more improved safety in unfit patients with diffuse large-B cell lymphoma compared to R-miniCHOP. BR should be considered as an alternative treatment in this subgroup of patients.

\section{Introduction}

Diffuse large B-cell lymphoma (DLBCL) is the most common type of non-Hodgkin lymphoma, with $40 \%$ incidence in patients age over 70 years old [1]. The chimeric anti-CD20 monoclonal antibody rituximab plus $\mathrm{CHOP}$ (cyclophosphamide, doxorubicin, vincristine, and prednisone) chemotherapy (R-CHOP) has dramatically improved the overall survival in patients with DLBCL in the last two decades. R-CHOP was recommended as a standard treatment for patients with DLBCL by the Groupe d'Etudes des Lymphomes de l'Adulte (GELA), depending on patients' age. Full dose R-CHOP was initially preferred in adult patients and elderly patients aged 60-80 [2, 3]. An attenuated immunochemotherapy regimen, R-miniCHOP, was identified and showed favorable efficacy and safety in patients over 80 years old [4]. However, the presence of comorbidities and concomitant infections often led to reduced tolerability to treatmentrelated toxicities, resulting in treatment discontinuation and treatment failure in older patients [5].

In lymphoma, the cut-off age 65 was defined as a watershed between younger and older patients [6]. The $\mathrm{R}$-miniCHOP regimen recommended by GELA for the treatment of DLBCL is also based on whether the patients is 80 years or older. Nevertheless, treatment should be individualized while incorporating individual life expectancy, functional reserve and social support into consideration [7]. A complete comprehensive geriatric assessment (CGA) should be undertaken to determine if older patients can 
tolerate intensive therapy with curative intent. CGA divides older patients into three categories: fit, unfit and frail, according to age, comorbidities and functional abilities of daily living [8]. CGA has proved to be an efficacy tool to identify fit patients who can benefit from intensive curative approach [9]. To date, there are few studies on therapeutic regimens applied in unfit patients except R-miniCHOP. Previously, studies had shown that treatment failure of R-miniCHOP in older patients $(>80)$ with DLBCL was associated with treatment-related toxicities and pre-existing medical comorbidities other than advanced age and relatively-low dose intensity [5]. Thus, more efficacious and low-toxicity regimens need to be exploited.

Bendamustine is a unique bifunctional alkylating agent with antimetabolic properties and antitumor effects [10]. The rebirth of bendamustine was in that it exhibited synergistic antitumor effects combined with rituximab (BR) for the treatment of lymphoid malignancies [11, 12]. Previous clinical trials reported that BR improved survival outcome in indolent non-Hodgkin lymphoma (NHL) and relapsed/refractory DLBCL patients $[13,14]$. There are currently limited clinical data available to rationalize therapeutic regimens for unfit patients with newly diagnosed DLBCL. A prospective phase II study showed anti-CD20 monoclonal antibody obinutuzumab plus CHOP was efficacious and safe for treating unfit patients with DLBCL [15]. Recently, a phase II clinical trial demonstrated that the BR regimen was a promising first-line treatment for frail patients with DLBCL due to its promising activity and manageable toxicity profile [16].

There is no prospective study comparing $\mathrm{BR}$ and $\mathrm{R}$-miniCHOP as frontline treatment in unfit patients at present. Our study investigated the efficacy, safety, toxicity profile, and overall remission rate (ORR) of BR compared to R-miniCHOP as first-line treatment in unfit patients with newly diagnosed DLBCL or follicular lymphoma (FL) grade 3B in China.

\section{Materials And Methods}

\section{Study design and patient eligibility}

FL grade $3 \mathrm{~B}$ is closely related to DLBCL and may transform to DLBCL. Therefore, the standard treatment regimens used in FL grade 3B are similar to that of DLBCL in routine clinical practice [17]. In this study, patients newly diagnosed with DLBCL or FL grade 3B were enrolled from January 2020 to December 2020. Diagnosis of DLBCL or FL grade 3B based on histopathologic morphology, immunohistology and clinical feature recommended by WHO 2016 [18].

Patients were classified as unfit according to simple CGA. Table 1 illustrates the inclusion criteria for this study [9]. Patients considered to be unfit included in this study met the following criteria: (a) Age $\geq 70$; (b) White blood cells $\geq 3.0 \times 10^{9} / \mathrm{L}$ or granulocytes $\geq 1.5 \times 10^{9} / \mathrm{L}$, hemoglobin $\geq 90.0 \times 10^{12} / \mathrm{L}$, platelets $\geq 100 \times 10^{9} / \mathrm{L}$; (c) Normal sinus rhythm, ejection fraction $50-70 \%$ on echocardiography; (d) ALT and AST levels below the $2 \times$ upper limit of normal, serum albumin $\geq 30.0 \mathrm{~g} / \mathrm{L}$, serum creatinine below the 1.5xupper limit of normal; (e) HIV/AIDS negative.

Clinical staging was based on modified Lugano 2014 staging criteria. The risk stratification was identified using International Prognostic Index (IPI). All the patients were received at least 2 cycles of R- 
miniCHOP or BR, depending on patients' performance status and the presence of comorbidities. Clinical features of R-miniCHOP and BR regimens are summarized in Table 2. In patients presenting with potential worsening cardiac function, doxorubicin was replaced by the liposome Adriamycin.

This study was approved by the Ethics and Scientific Committee of Union Hospital of Tongji Medical College, Huazhong University of Science and Technology with approval number 2020211. All the patients were given written informed consent before immunochemotherapy and the data were collected from electronic medical record of Union hospital, Tongji Medical college.

\section{Response evaluation}

Treatment responses were evaluated by computed tomography scans or positron emission tomography (PET) scan after at least 2-cycles of immunochemotherapy. Bone marrow aspiration and immunotyping were also routinely performed to determine bone marrow invasion at initial diagnosis. Therapeutic evaluation based on Lugano 2014 classification and divided into imaging remission and metabolic remission, including complete remission (CR), partial remission (PR), stable disease (SD) and progressive disease (PD) [19].

\section{Adverse reactions assessment}

Routine physical examination, hematological and biochemistry tests, electrocardiogram and electrocardiograph, were done before and after every cycle of immunochemotherapy. Adverse effects were graded according to the National Cancer Institute Common Terminology Criteria for Adverse Events version $4.0[20]$.

\section{Statistical analysis}

The laboratory and clinical date, response rates and adverse reactions between R-miniCHOP and BR groups were analyzed by using chi-square test and Mann Whitney $U$ test. Statistical analyses were performed by using GraphPad 7.0 and the Statistical Package for the Social Sciences version 24.0. Pvalue $<0.05$ was regarded as statistically significant.

\section{Results}

\section{Baseline characteristics of patients}

Thirty-two patients were included from Union hospital of Wuhan and Xiangyang No.1 hospital between January 2020 and November 2020. The median age was 74 years (range 70 to 82), and the male to female ratio was 0.88:1. Twenty-five patients were diagnosed as DLBCL, seven patients with FL grade 3B. Eighteen patients (56.25\%) were diagnosed at Stage IV. BR regimen was selected when patients presented with with medical comorbidities. Twenty patients were treated with R-miniCHOP, and twelve patients received BR instead. There were no significance in Age, Sex, Stage between two groups. The baseline characteristics are listed in Table 3. 


\section{Treatment and Response}

Two patients received 2 cycles of R-miniCHOP and one patient received 2 cycles of BR because of tumor progression and severe infection caused by myelosuppression. Three patients received 3-cycles of BR before this study was terminated. The rest of patients received 4-6 cycles of immunochemotherapy. The percentage CR rate was similar between both groups ( $35 \%$ vs $33.3 \%$ ). The objective remission rate (ORR) in the BR group was minimally improved compared to the R-miniCHOP group (83.3\% vs $75 \%$ ), but with no statistical significance $(P=0.581)$ as shown in Table. 4 .

\section{Adverse reactions}

Adverse events were classified as hematological and non-hematologic adverse reactions (Table 5). The RminiCHOP group had a higher incidence of leukopenia $(P=0.029)$ compared to the BR group. Furthermore, six patients were reported to have an occurrence of infection, and four patients reported with cardiac events in the R-miniCHOP group. However, the BR group had a higher incidence of transient fever $(P=0.036)$.

\section{Sensitivity analysis}

Univariate analyses were performed to clarify the relationship between prognostic factors and ORR of the 32 patients. In the univariate analysis, ECOG 0-1, no extranodal sites, LDH and ESR level, tumor mass $<10$ $\mathrm{cm}$ were unrelated with ORR. However, $\beta 2$-microglobulin $<3.0 \mathrm{mg} / \mathrm{L}$ maybe predictive of higher ORR $(P=$ 0.022) (Table. S1).

\section{Discussion}

The Surveillance, Epidemiology and End Results (SEER) program predicted that the incidence of NHL would be estimated at around $4 \%$ of all cancer cases worldwide in 2024, with a median age at 66 years [21]. DLBCL accounts for about $31 \%$ of $\mathrm{NHL}$ cases, with the majority of DLBCL patients being over 60 years [6,22]. Therefore, measures to improve patient outcomes, individual life expectancy, and survival for this category of patients must be enhanced.

In the recent past, $\mathrm{R}-\mathrm{CHOP}$ was the recommended standard regimen for the treatment DLBCL in older patients below the age of 80 years. Older patients aged 60-80 years with DLBCL could achieve a relatively high $\mathrm{CR}$ rate and prolonged median survival after 4-6 cycles of R-CHOP [3]. R-miniCHOP offered an improved comprise between safety and efficiency in patients aged 80 years and older as a substantial number of older people could be cured [4]. However, complications and adverse treatment reactions were reported to increase in patients aged 70 years and older [23]. Several risk factors, including comorbidity, functional impairment, cognitive decline, poor performance score and social isolation, contributed to the treatment-related toxicity [24]. Despite having R-CHOP or R-miniCHOP as treatment options in older patients, the presence of comorbidities and treatment-related toxicity, to some extent, contributes to 
limited available therapeutic options in real-world practice. Thus, promising regimens need to be exploited and in order to provide an optimal balance between efficiency and safety in older patients.

Several studies demonstrated that the BR regimen was a promising prospect in both indolent and aggressive lymphomas because of its modest activity and manageable toxicity profile. The BRIGHT study reported that the BR regimen had a better long-term disease control than R-CHOP regimen and therefore, should be recommended as the first-line treatment in indolent and mantel-cell lymphoma [25]. Furthermore, a multicenter, retrospective study reported that the BR regimen was less toxic and more efficient compared to the $\mathrm{R}-\mathrm{CHOP}$ regimen in patients with $\mathrm{FL}$ grade $3 \mathrm{~A}$ [26]. BR regimen demonstrated a low rate of nonhematologic adverse events in older patients with chronic lymphocytic leukemia [27]. Moreover, BR regimen was well-tolerated and safety in indolent NHL patients with renal impairment [28].

In 2011, an exploratory clinical study suggested that BR may be an alternative treatment for aggressive lymphomas in older patients 80 years and older ineligible for R-CHOP therapy [29]. To date, a few studies have demonstrated BR was a feasible option for the first-line treatment of DLBCL in elderly patients [16, 30-32]. Table 6 illustrates the different ranges of ORR, CRR and median PFS in BR groups; ORR 50-83.3\%, CRR 20-54\% and median progression-free survival ranged 7.7-33 months. On the contrary, results obtained by the GELA analysis, ORR in the R-miniCHOP group was 75\% [4]. BR showed nearly equivalent efficacy in this category of patients. Our study further verified the findings of the GELA analysis, the ORR was $83.3 \%$ and $75 \%$ in the BR group and R-miniCHOP group, respectively, with no statistical significance. Furthermore, in 32 patients, $\beta 2$-microglobulin $<3.0 \mathrm{mg} / \mathrm{L}$ was predictive of higher ORR in our univariate analyses of prognostic factors.

In the previous study, nausea and vomiting were frequently reported in older patients after BR treatment. This adverse reaction was equally commonly reported in our study (50\%) [26]. However, it was not of significance compared to R-miniCHOP group $(P=0.581)$. Importantly, the BR group had a lower rate of leukopenia compared to the R-miniCHOP group. This result showed that BR is likely to reduce the risk of infection and febrile neutropenia. Additionally, BR had a relative low rate of cardiac events due to the absence of anthracyclines, showing that BR is safer and tolerable for unfit older patients. It's worth noting that BR had a higher incidence of transient fever which could be attributed to drug-induced fever as the inflammatory indicators such as $\mathrm{C}$-reaction protein and procalcitonin maintained within normal range. Generally, the body temperature could be quickly reduced to normal after auxiliary antipyretic treatment. However, when this symptom arises at the initiation of treatment, it is difficult for physicians to determine the cause of fever which may preclude treatment.

The limitation of our study was that it was a small cohort size and short follow-up (eleven month) duration study. Bendamustine was brought to market in China on May 2019. So far, it was only used in part of first-ties cities. Furthermore, it is relatively expensive with the cost of bendamustine not being reimbursed by the national health insurance system in China. The number of elderly patients newly diagnosed with DLBCL and defined as unfit was small. Thus, it is a challenge to recruit enough unfit participants in such a short time. that was also the reason why overall survival and median progression- 
free survival were not analyzed in our study. For this reason, our study could not analyze overall survival and median progression-free survival. We believe that a large-scale and long-term follow-up prospective study will provide better insight into the future.

The emergence of new targeted drugs also provides more options for the treatment of DLBCL. A recent clinical trial showed that the anti-CD79b antibody polatuzumab vedotin combined with BR could reduce the risk of death in relapsed/refractory DLBCL [33]. The novel histone deacetylases inhibitor chidamide could synergize with rituximab by upregulating CD20 expression in DLBCL and significantly inhibited the tumor growth in vitro and in vivo [34]. The phosphoinositide 3-kinase (PI3K) inhibitor copanlisib showed high cytotoxicity in vivo and could improve survival in DLBCL model [35]. Moreover, the Bcl-2 inhibitor venetoclax and Bruton's tyrosine kinase inhibitor ibrutinib could enhancing the sensitivity of PI3K inhibitor in actived B-cell like DLBCL $[35,36]$. Thus, more clinical trials should be conducted to explore new therapeutic regimens for DLBCL.

\section{Conclusion}

Currently, the treatment for unfit older patients with DLBCL remains a daunting challenge for physicians. The choice of treatment should be individualized, with an accurate assessment of the risk-benefit ratio undertaken for each patient before treatment. As such, considering our results, BR is a promising regimen with lesser toxicity and higher efficacy for older patients with DLBCL. Therefore, BR is recommended as an alternative regimen of $\mathrm{R}$-miniCHOP and a first-line regimen in unfit patients with DLBCL.

\section{Declarations}

\section{Funding}

This study was supported by grant from the National Natural Science Foundation of China (No. 81672940) and the Clinical Research Physician Program of Tongji Medical College, Huazhong University of Science and Technology (No.5001530053).

\section{Authors' contributions}

YHD and LLZ provided the direction of study. DDZ collected and analyzed the data, wrote the manuscript, NMK revised the manuscript. All authors read and approved the final manuscript.

\section{Conflict of interests}

The authors declare that they have no competing interests.

\section{Data availability statement}

The clinical data supporting the conclusions of this manuscript will be made available by the authors. 


\section{References}

1. d'Amore F, Brincker $H$, Christensen BE, Thorling K, Pedersen M, Nielsen JL, et al. Non-Hodgkin's lymphoma in the elderly. A study of 602 patients aged 70 or older from a Danish population-based registry. The Danish LYEO-Study Group. Annals of oncology : official journal of the European Society for Medical Oncology. 1992; 3: 379-86.

2. Feugier $P$, Van Hoof $A$, Sebban $C$, Solal-Celigny $P$, Bouabdallah R, Fermé $C$, et al. Long-term results of the R-CHOP study in the treatment of elderly patients with diffuse large B-cell lymphoma: a study by the Groupe d'Etude des Lymphomes de l'Adulte. Journal of clinical oncology : official journal of the American Society of Clinical Oncology. 2005; 23: 4117-26.

3. Coiffier B, Thieblemont C, Van Den Neste E, Lepeu G, Plantier I, Castaigne S, et al. Long-term outcome of patients in the $\mathrm{LNH}-98.5$ trial, the first randomized study comparing rituximab-CHOP to standard CHOP chemotherapy in DLBCL patients: a study by the Groupe d'Etudes des Lymphomes de l'Adulte. Blood. 2010; 116: 2040-5.

4. Peyrade F, Jardin F, Thieblemont C, Thyss A, Emile JF, Castaigne S, et al. Attenuated immunochemotherapy regimen (R-miniCHOP) in elderly patients older than 80 years with diffuse large B-cell lymphoma: a multicentre, single-arm, phase 2 trial. The Lancet Oncology. 2011; 12: 460-8.

5. Kobayashi Y, Miura K, Hojo A, Hatta Y, Tanaka T, Kurita D, et al. Charlson Comorbidity Index is an independent prognostic factor among elderly patients with diffuse large B-cell lymphoma. Journal of cancer research and clinical oncology. 2011; 137: 1079-84.

6. Fields PA, Linch DC. Treatment of the elderly patient with diffuse large B cell lymphoma. British journal of haematology. 2012; 157: 159-70.

7. Balducci L, Extermann M. Management of cancer in the older person: a practical approach. The oncologist. 2000; 5: 224-37.

8. Extermann M, Aapro M, Bernabei R, Cohen HJ, Droz JP, Lichtman S, et al. Use of comprehensive geriatric assessment in older cancer patients: recommendations from the task force on CGA of the International Society of Geriatric Oncology (SIOG). Critical reviews in oncology/hematology. 2005; 55: 241-52.

9. Tucci A, Martelli M, Rigacci L, Riccomagno P, Cabras MG, Salvi F, et al. Comprehensive geriatric assessment is an essential tool to support treatment decisions in elderly patients with diffuse large B-cell lymphoma: a prospective multicenter evaluation in 173 patients by the Lymphoma Italian Foundation (FIL). Leukemia \& lymphoma. 2015; 56: 921-6.

10. Cheson BD, Rummel MJ. Bendamustine: rebirth of an old drug. Journal of clinical oncology : official journal of the American Society of Clinical Oncology. 2009; 27: 1492-501.

11. Rummel MJ, Niederle N, Maschmeyer G, Banat GA, von Grünhagen U, Losem C, et al. Bendamustine plus rituximab versus $\mathrm{CHOP}$ plus rituximab as first-line treatment for patients with indolent and mantle-cell lymphomas: an open-label, multicentre, randomised, phase 3 non-inferiority trial. Lancet (London, England). 2013; 381: 1203-10. 
12. Flinn IW, van der Jagt R, Kahl BS, Wood P, Hawkins TE, Macdonald D, et al. Randomized trial of bendamustine-rituximab or R-CHOP/R-CVP in first-line treatment of indolent NHL or MCL: the BRIGHT study. Blood. 2014; 123: 2944-52.

13. Hong JY, Yoon DH, Suh C, Kim WS, Kim SJ, Jo JC, et al. Bendamustine plus rituximab for relapsed or refractory diffuse large $B$ cell lymphoma: a multicenter retrospective analysis. Annals of hematology. 2018; 97: 1437-43.

14. Ohmachi K, Niitsu N, Uchida T, Kim SJ, Ando K, Takahashi N, et al. Multicenter phase II study of bendamustine plus rituximab in patients with relapsed or refractory diffuse large B-cell lymphoma. Journal of clinical oncology : official journal of the American Society of Clinical Oncology. 2013; 31: 2103-9.

15. Merli F, Cavallo F, Salvi F, Tucci A, Musuraca G, Nassi L, et al. Obinutuzumab and miniCHOP for unfit patients with diffuse large B-cell lymphoma. A phase II study by Fondazione Italiana Linfomi. Journal of geriatric oncology. 2020; 11: 37-40.

16. Storti S, Spina M, Pesce EA, Salvi F, Merli M, Ruffini A, et al. Rituximab plus bendamustine as frontline treatment in frail elderly (>70 years) patients with diffuse large B-cell non-Hodgkin lymphoma: a phase II multicenter study of the Fondazione Italiana Linfomi. Haematologica. 2018; 103: 1345-50.

17. Horn H, Kohler C, Witzig R, Kreuz M, Leich E, Klapper W, et al. Gene expression profiling reveals a close relationship between follicular lymphoma grade $3 \mathrm{~A}$ and $3 \mathrm{~B}$, but distinct profiles of follicular lymphoma grade 1 and 2. Haematologica. 2018; 103: 1182-90.

18. Cazzola M. Introduction to a review series: the 2016 revision of the WHO classification of tumors of hematopoietic and lymphoid tissues. Blood. 2016; 127: 2361-4.

19. Cheson BD, Fisher RI, Barrington SF, Cavalli F, Schwartz LH, Zucca E, et al. Recommendations for initial evaluation, staging, and response assessment of Hodgkin and non-Hodgkin lymphoma: the Lugano classification. Journal of clinical oncology : official journal of the American Society of Clinical Oncology. 2014; 32: 3059-68.

20. Dueck AC, Mendoza TR, Mitchell SA, Reeve BB, Castro KM, Rogak LJ, et al. Validity and Reliability of the US National Cancer Institute's Patient-Reported Outcomes Version of the Common Terminology Criteria for Adverse Events (PRO-CTCAE). JAMA oncology. 2015; 1: 1051-9.

21. DeSantis CE, Lin CC, Mariotto AB, Siegel RL, Stein KD, Kramer JL, et al. Cancer treatment and survivorship statistics, 2014. CA: a cancer journal for clinicians. 2014; 64: 252-71.

22. Martelli M, Ferreri AJ, Agostinelli C, Di Rocco A, Pfreundschuh M, Pileri SA. Diffuse large B-cell lymphoma. Critical reviews in oncology/hematology. 2013; 87: 146-71.

23. Advani RH, Chen H, Habermann TM, Morrison VA, Weller EA, Fisher RI, et al. Comparison of conventional prognostic indices in patients older than 60 years with diffuse large B-cell lymphoma treated with R-CHOP in the US Intergroup Study (ECOG 4494, CALGB 9793): consideration of age greater than 70 years in an elderly prognostic index (E-IPI). British journal of haematology. 2010; 151: 143-51. 
24. Pal SK, Katheria V, Hurria A. Evaluating the older patient with cancer: understanding frailty and the geriatric assessment. CA: a cancer journal for clinicians. 2010; 60: 120-32.

25. Flinn IW, van der Jagt R, Kahl B, Wood P, Hawkins T, MacDonald D, et al. First-Line Treatment of Patients With Indolent Non-Hodgkin Lymphoma or Mantle-Cell Lymphoma With Bendamustine Plus Rituximab Versus R-CHOP or R-CVP: Results of the BRIGHT 5-Year Follow-Up Study. Journal of clinical oncology : official journal of the American Society of Clinical Oncology. 2019; 37: 984-91.

26. Mondello P, Steiner N, Willenbacher W, Cerchione C, Nappi D, Mauro E, et al. Bendamustine plus Rituximab Versus R-CHOP as First-Line Treatment for Patients with Follicular Lymphoma Grade 3A: Evidence from a Multicenter, Retrospective Study. The oncologist. 2018; 23: 454-60.

27. Woyach JA, Ruppert AS, Heerema NA, Zhao W, Booth AM, Ding W, et al. Ibrutinib Regimens versus Chemoimmunotherapy in Older Patients with Untreated CLL. The New England journal of medicine. 2018; 379: 2517-28.

28. Ribes D, Hachem HEL, Oberic L, Vergez F, Delas A, Belliere J, et al. Bendamustine plus rituximab for indolent B-cell lymphoma of renal significance. American journal of hematology. 2018; 93: 356-62.

29. Weidmann E, Neumann A, Fauth F, Atmaca A, Al-Batran SE, Pauligk C, et al. Phase II study of bendamustine in combination with rituximab as first-line treatment in patients 80 years or older with aggressive B-cell lymphomas. Annals of oncology : official journal of the European Society for Medical Oncology. 2011; 22: 1839-44.

30. Horn J, Kleber M, Hieke S, Schmitt-Gräff A, Wäsch R, Engelhardt M. Treatment option of bendamustine in combination with rituximab in elderly and frail patients with aggressive B-nonHodgkin lymphoma: rational, efficacy, and tolerance. Annals of hematology. 2012; 91: 1579-86.

31. Cheng CL, Liu JH, Chou SC, Yao M, Tang JL, Tien HF. Retrospective analysis of frontline treatment efficacy in elderly patients with diffuse large B-cell lymphoma. European journal of haematology. 2018; 101: 28-37.

32. Park SI, Grover NS, Olajide O, Asch AS, Wall JG, Richards KL, et al. A phase II trial of bendamustine in combination with rituximab in older patients with previously untreated diffuse large B-cell lymphoma. British journal of haematology. 2016; 175: 281-9.

33. Sehn LH, Herrera AF, Flowers CR, Kamdar MK, McMillan A, Hertzberg M, et al. Polatuzumab Vedotin in Relapsed or Refractory Diffuse Large B-Cell Lymphoma. Journal of clinical oncology : official journal of the American Society of Clinical Oncology. 2020; 38: 155-65.

34. Guan XW, Wang HQ, Ban WW, Chang Z, Chen HZ, Jia L, et al. Novel HDAC inhibitor Chidamide synergizes with Rituximab to inhibit diffuse large B-cell lymphoma tumour growth by upregulating CD20. Cell death \& disease. 2020; 11: 20.

35. Bojarczuk K, Wienand K, Ryan JA, Chen L, Villalobos-Ortiz M, Mandato E, et al. Targeted inhibition of $\mathrm{PI} 3 \mathrm{Ka} / \delta$ is synergistic with BCL-2 blockade in genetically defined subtypes of DLBCL. Blood. 2019; 133: $70-80$.

36. Sasi BK, Martines C, Xerxa E, Porro F, Kalkan H, Fazio R, et al. Inhibition of SYK or BTK augments venetoclax sensitivity in SHP1-negative/BCL-2-positive diffuse large B-cell lymphoma. Leukemia. 


\section{Tables}

Table 1. Definition of unfit according to CGA

\begin{tabular}{|cllll|}
\hline & Age & ADL score & IADL score & CIRS- \\
\hline$>79$ & 6 & 8 & no grade-3/4 comorbidities \\
& & & & and $<5$ grade-2 comorbidities \\
Unfit & & & $6-7$ & no grade-3/4 comorbidities \\
& & & & and $5-8$ grade-2 comorbidities \\
\hline
\end{tabular}

Note: $A D L$, activity of daily living; IADL, instrumental activity of daily living; CIRS-, Cumulative IIIness Rating Score for Geriatrics; CGA, comprehensive geriatric assessment.

Table 2. The R-miniCHOP and BR rigimens

\begin{tabular}{|llll|}
\hline Agents & Does & Route & Time \\
R-miniCHOP & & & \\
Rituximab & $375 \mathrm{mg} / \mathrm{m}^{2}$ & IV & Day 1 \\
Cyclophosphamide & $375-562.5 \mathrm{mg} / \mathrm{m}^{2}$ & IV & Day 2 \\
Vincristine & $1.4 \mathrm{mg} / \mathrm{m}^{2} \otimes_{\max } \leq 2 \mathrm{mg} \rrbracket$ & IV & Day 2 \\
Vindesine & $2-3 \mathrm{mg} / \mathrm{m}^{2}\left(\mathrm{D}_{\max } \leq 3 \mathrm{mg}\right)$ & & \\
Doxorubicin & $25-35 \mathrm{mg} / \mathrm{m}^{2}$ & IV & Day 2 \\
(liposome adriamycin) & $\left(10-15 \mathrm{mg} / \mathrm{m}^{2}\right)$ & & \\
Prednisone & $100 \mathrm{mg}$ & PO & Day $1-5$ \\
BR & & & \\
Rituximab & $375 \mathrm{mg} / \mathrm{m}^{2}$ & IV & Day 1 \\
\hline Bendamustine & $60-90 \mathrm{mg} / \mathrm{m}^{2}$ & IV & Day 2-3 \\
\hline
\end{tabular}

Note: Both R-miniCHOP and BR regimen were repeated every 21 days. When elderly patients appeared with cardiac insufficiency, doxorubicin were often replaced with liposome adriamycin. IV, intravenously; PO, per os. 
Table 3. Characteristics of patients enrolled in the study

\begin{tabular}{|c|c|c|c|}
\hline \multirow[t]{3}{*}{ Variables } & \multicolumn{2}{|c|}{ Number of patients $(n=32)$} & \multirow[t]{3}{*}{$P$} \\
\hline & R-miniCHOP & BR & \\
\hline & $(n=20)$ & $(n=12)$ & \\
\hline Age & & & 0.273 \\
\hline Range & $70-81$ & $70-82$ & \\
\hline Median & 73 & 76 & \\
\hline$\geq 70,<80$ & 19 & 10 & \\
\hline$>80$ & 1 & 2 & \\
\hline Sex & & & 0.647 \\
\hline Male & 10 & 5 & \\
\hline Female & 10 & 7 & \\
\hline Pathology & & & 0.740 \\
\hline$F L$, grade $3 B$ & 4 & 3 & \\
\hline DLBCL & 16 & 9 & \\
\hline Stage (Lugano) & & & 0.991 \\
\hline प & 3 & 2 & \\
\hline प & 5 & 3 & \\
\hline प & 2 & 1 & \\
\hline प & 12 & 6 & \\
\hline IPI & & & 0.241 \\
\hline 1 & 2 & 4 & \\
\hline 2 & 6 & 2 & \\
\hline 3 or more & 12 & 6 & \\
\hline ECOG & & & 0.043 \\
\hline 0 & 2 & 1 & \\
\hline 1 & 7 & 4 & \\
\hline 2 & 11 & 7 & \\
\hline
\end{tabular}


Note: $P$, Chi-square. Abbreviations: IPI, International Prognostic Index; ECOG: Eastern Cooperative Oncology Group Performance Status.

Table 4. Overall response rates of R-miniCHOP and BR regimens

\begin{tabular}{|llll|}
\hline Response & R-miniCHOP $(\mathbf{n}=20)$ & BR (n=12) & P value \\
\hline Complete remission rate & $35 \%(7 / 20)$ & $33.3 \%(4 / 12)$ & 0.923 \\
\hline Partial remission rate & $40 \%(8 / 20)$ & $50 \%(6 / 12)$ & 0.580 \\
\hline Stable disease rate & $10 \%(2 / 20)$ & $8.3 \%(1 / 12)$ & 0.875 \\
\hline Progressive disease rate & $15 \%(3 / 20)$ & $8.3 \%(1 / 12)$ & 0.581 \\
\hline Objective remission rate & $75 \%(15 / 20)$ & $83.3 \%(10 / 12)$ & 0.581 \\
\hline
\end{tabular}

Table 5. Hematological and extra-hematological adverse events between R-miniCHOP and BR groups.

\begin{tabular}{|llll|}
\hline Toxicity & \multicolumn{2}{l}{ Grade of adverse reaction } & \\
\cline { 2 - 3 } & \multicolumn{1}{l}{ R-miniCHOP $(\mathbf{n}=20)$} & BR (n=12) & P value \\
\hline Hematologic & & & \\
\hline Leukopenia & $12(60 \%)$ & $3(25 \%)$ & $0.029 *$ \\
\hline Anemia & $13(65 \%)$ & $6(50 \%)$ & 0.403 \\
\hline Thrombocytopenia & $6(30 \%)$ & $2(17 \%)$ & 0.399 \\
\hline Non-hematologic & & & \\
\hline Nausea and vomiting & $8(40 \%)$ & $6(50 \%)$ & 0.581 \\
\hline ALT/AST elevation & $6(30 \%)$ & $2(17 \%)$ & 0.399 \\
\hline Hypoalbuminemia & $3(15 \%)$ & $2(17 \%)$ & 0.900 \\
\hline Cardiac disorders & $4(20 \%)$ & $1(8 \%)$ & 0.378 \\
\hline Nervous system disorders & $3(15 \%)$ & $1(8 \%)$ & 0.580 \\
\hline Allergy & $5(25 \%)$ & $2(17 \%)$ & 0.581 \\
\hline Transient fever & $2(10 \%)$ & $5(42 \%)$ & $0.036 *$ \\
\hline Electrolyte imbalance & $8(40 \%)$ & $7(58 \%)$ & 0.314 \\
\hline Infection & $6(30 \%)$ & $1(8 \%)$ & 0.151 \\
\hline
\end{tabular}


Table 6. Summary of prospective studies of BR as frontline treatment in elderly patients with newly diagnosed with DLBCL.

\begin{tabular}{|c|c|c|c|c|c|c|c|}
\hline Author and Year & Region & Patients & Age & Bendamustine & ORR\% & CRR\% & mPFS \\
\hline $\begin{array}{l}\text { Weidmann et al, } \\
2011\end{array}$ & Germany & 13 & $\begin{array}{l}85(80- \\
95)\end{array}$ & $\begin{array}{l}120 \\
\mathrm{mg} / \mathrm{m}^{2} / \mathrm{q} 3 \mathrm{w}\end{array}$ & $69 \%$ & $54 \%$ & 7.7 \\
\hline Horn et al, 2012 & Germany & 20 & $\begin{array}{l}72(51- \\
86)\end{array}$ & $90 \mathrm{mg} / \mathrm{m}^{2} / \mathrm{q} 4 \mathrm{w}$ & $55 \%$ & $20 \%$ & 19.4 \\
\hline Park et al, 2016 & USA & 23 & $80(>65)$ & $\begin{array}{l}120 \\
\mathrm{mg} / \mathrm{m}^{2} / \mathrm{q} 3 \mathrm{w}\end{array}$ & $78 \%$ & $52 \%$ & 10.2 \\
\hline Storti et al, 2018 & Italy & 49 & $81(>70)$ & $90 \mathrm{mg} / \mathrm{m}^{2} / \mathrm{q} 4 \mathrm{w}$ & $62 \%$ & $53 \%$ & 33 \\
\hline Cheng et al, 2018 & Taiwan & 26 & $\begin{array}{l}81(75- \\
93)\end{array}$ & $90 \mathrm{mg} / \mathrm{m}^{2} / \mathrm{q} 3 \mathrm{w}$ & $50 \%$ & $42.3 \%$ & 11.2 \\
\hline $\begin{array}{l}\text { Current report, } \\
2020\end{array}$ & China & 12 & $\begin{array}{l}70(70- \\
82)\end{array}$ & $\begin{array}{l}60-90 \\
\mathrm{mg} / \mathrm{m}^{2} / \mathrm{q} 3 \mathrm{w}\end{array}$ & $85.3 \%$ & $33.3 \%$ & NA \\
\hline
\end{tabular}

Note: CRR: complete remission rate; ORR: overall response rate; mPFS: median progression-free survival; NA, not available

\section{Supplementary Files}

This is a list of supplementary files associated with this preprint. Click to download.

- TableS1.docx 